Journal for -... ImmunoTherapy of Cancer

\section{Modified Glasgow Prognostic Score associated with survival in metastatic renal cell carcinoma treated with immune checkpoint inhibitors}

To cite: Brown JT, Liu Y, Shabto JM, et al. Modified Glasgow Prognostic Score associated with survival in metastatic renal cell carcinoma treated with immune checkpoint inhibitors. Journal for ImmunoTherapy of Cancer 2021;9:e002851. doi:10.1136/ jitc-2021-002851

Accepted 24 May 2021

\section{Check for updates}

(c) Author(s) (or their employer(s)) 2021. Re-use permitted under CC BY-NC. No commercial re-use. See rights and permissions. Published by BMJ.

${ }^{1}$ Winship Cancer Institute of Emory University, Atlanta, Georgia, USA

${ }^{2}$ Department of Hematology and Medical Oncology, Emory University School of Medicine, Atlanta, Georgia, USA ${ }^{3}$ Departments of Biostatistics and Bioinformatics, Emory University, Atlanta, Georgia, USA ${ }^{4}$ Department of Pharmaceutical Services, Emory University, Atlanta, Georgia, USA

${ }^{5}$ Department of Urology, Emory University School of Medicine, Atlanta, Georgia, USA

Correspondence to Dr Mehmet Asim Bilen; mehmet.a.bilen@emory.edu

\section{ABSTRACT}

Background The modified Glasgow Prognostic Score (mGPS) is a composite biomarker that uses albumin and $\mathrm{C}$ reactive protein (CRP). There are multiple immune checkpoint inhibitor (ICI)-based combinations approved for metastatic renal cell carcinoma (mRCC). We investigated the ability of mGPS to predict outcomes in patients with mRCC receiving $\mathrm{ICl}$.

Methods We retrospectively reviewed patients with $\mathrm{mRCC}$ treated with $\mathrm{ICl}$ as monotherapy or in combination at Winship Cancer Institute between 2015 and 2020. Overall survival (OS) and progression-free survival (PFS) were measured from the start date of ICI until death or clinical/ radiographical progression, respectively. The baseline mGPS was defined as a summary score based on pre$\mathrm{ICl}$ values with one point given for $\mathrm{CRP}>10 \mathrm{mg} / \mathrm{L}$ and/or albumin $<3.5 \mathrm{~g} / \mathrm{dL}$, resulting in possible scores of 0,1 and 2. If only albumin was low with a normal CRP, no points were awarded. Univariate analysis (UVA) and multivariate analysis (MVA) were carried out using Cox proportional hazard model. Outcomes were also assessed by KaplanMeier analysis.

Results 156 patients were included with a median followup 24.2 months. The median age was 64 years and $78 \%$ had clear cell histology. Baseline mGPS was 0 in 36\%, 1 in $40 \%$ and 2 in $24 \%$ of patients. In UVA, a baseline mGPS of 2 was associated with shorter OS (HR $4.29,95 \% \mathrm{Cl}$ 2.24 to $8.24, \mathrm{p}<0.001)$ and PFS (HR 1.90, 95\% $\mathrm{Cl} 1.20$ to $3.01, p=0.006$ ) relative to a score of 0 ; this disparity in outcome based on baseline mGPS persisted in MVA. The respective median $0 S$ of patients with baseline mGPS of 0,1 and 2 was 44.5 (95\% Cl 27.3 to not evaluable), 15.3 (95\% Cl 11.0 to 24.2$)$ and 10 (95\% Cl 4.6 to 17.5$)$ months $(p<0.0001)$. The median PFS of these three cohorts was 6.7 (95\% Cl 3.6 to 13.1$), 4.2$ (95\% Cl 2.9 to 6.2 ) and $2.6(95 \% \mathrm{Cl} 2.0$ to 5.6$)$, respectively $(\mathrm{p}=0.0216)$. The discrimination power of baseline mGPS to predict survival outcomes was comparable to the IMDC risk score based on Uno's c-statistic (OS: 0.6312 vs 0.6102 , PFS: 0.5752 vs 0.5533).

Conclusion The mGPS is prognostic in this cohort of patients with $\mathrm{mRCC}$ treated with $\mathrm{ICl}$ as monotherapy or in combination. These results warrant external and prospective validation.

\section{INTRODUCTION}

Kidney cancer was diagnosed in 73,750 people in the USA and was responsible for approximately 13,830 deaths in 2020. ${ }^{1}$ Renal cell carcinoma (RCC) comprises approximately $85 \%$ of primary cancers of the kidney with clear cell histology making up 75\%-85\% of RCC. ${ }^{2}$ While the mainstay of therapy for localized disease is surgery, the $16 \%$ of patients who present with de novo metastatic disease and approximately half of the patients with locally advanced disease that recur after definitive surgery are treated with systemic therapy. This cohort with metastatic renal cell carcinoma (mRCC) has a 5-year survival rate of $13 \%$ based on population-level data from 2010 to 2016, although newer therapies are poised to improve outcomes. ${ }^{3}$

Several risk models exist to prognosticate and direct first-line therapy. The Memorial Sloan Kettering Cancer Center (MSKCG) prognostic model incorporates time from diagnosis to treatment, Karnofsky performance status (KPS), serum lactate dehydrogenase $(\mathrm{LDH})$, calcium and hemoglobin, whereas the more widely used International Metastatic Renal Cell Carcinoma Database Consortium (IMDC) criteria replaces $\mathrm{LDH}$ with serum neutrophil and platelet count. ${ }^{45}$ These risk scores have been used in clinical trials to risk stratify patients and have also been incorporated into the approved indications for new therapies. However, the IMDC criteria were developed based on survival data from patients treated with antiangiogenic therapies including sunitinib, sorafenib 
and bevacizumab. The MSKCC model was derived from patients being treated with first-line interferon alfa-2a. Notably, neither prognostic model includes patients treated with contemporary immunotherapy, most commonly immune checkpoint inhibitors (ICIs) but also high-dose interleukin (IL)-2 in rare circumstances. Given our current practice, exploration of alternative and applicable prognostic tools is needed. Approved and commonly used in the first-line setting for clear cell histologies are the combinations of pembrolizumab and axitinib, pembrolizumab and lenvatinib, ipilimumab and nivolumab, axitinib and avelumab, and most recently, nivolumab and cabozantinib. ${ }^{6-10}$ The ubiquitous presence of ICI in the treatment paradigm for mRCC warrants consideration of a prognostic score capable of predicting outcomes in patients treated specifically with immunotherapy.

Systemic inflammation has been identified as a contributor to resistance to ICI across multiple malignancies including RCC. ${ }^{11-15}$ The original Glasgow Prognostic Score was developed by McMillan et al as a prognostic score in metastatic non-small cell lung cancer (NSCLC) and is based on inflammatory biomarkers; a point is assigned for a $\mathrm{C}$ reactive protein (CRP) of $>10 \mathrm{mg} / \mathrm{L}$ and/ or an albumin of $<3.5 \mathrm{~g} / \mathrm{dL}$ culminating in stratification of patients into low ( 0 points), intermediate (one point) and high (two points) risk. ${ }^{16}$ The score's prognostic capabilities were improved on by the modified Glasgow Prognostic Score (mGPS), which differs in that a point is only awarded for a low albumin if the CRP is elevated, thus more heavily weighting the inflammatory component of the score. ${ }^{17}$ The mGPS has been validated in nonmetastatic clear cell RCC treated with nephrectomy ${ }^{18-20}$ and more recently in a small cohort of patients with metastatic disease treated with nivolumab monotherapy in the second-line setting after targeted therapy. ${ }^{21}$ We sought to investigate its prognostic significance more broadly in patients with mRCC treated with immunotherapy in any line of therapy.

\section{PATIENTS AND METHODS}

We conducted a retrospective analysis of 156 patients with biopsy-proven mRCC. Included were those who received ICI in the form of an antiprogrammed cell death protein 1 (PD-1) or anti-programmed cell death protein ligand 1 (PD-L1) monoclonal antibody, either as monotherapy or in combination with an antiangiogenic agent, antiCTLA4 therapy, or experimental therapy in any line of treatment at the Winship Cancer Institute of Emory University between the years of 2015 and 2020. Patients were identified through a drug administration pharmacy database. Demographic and clinical data including age, sex, race, smoking status, Eastern Cooperative Oncology Group (ECOG) performance status, tumor histology, sites of metastatic disease, body mass index (BMI), baseline laboratory data within 2 weeks of receiving treatment with ICI as well as treatment course and response were extracted from the electronic medical record. Baseline laboratory values collected included albumin and CRP.

The primary outcomes assessed include progressionfree survival (PFS), overall survival (OS) and clinical benefit rate (CBR). PFS was defined as the duration of time in months between ICI initiation and clinical or radiographical progression or death and OS as the time between ICI initiation and death. Radiographical response and progression were defined using the Response Evaluation Criteria in Solid Tumors (RECIST) V.1.1. Univariate (UVA) and multivariate (MVA) analyses were undertaken using Cox proportional hazard model for OS and PFS. In MVA, the model was fitted followed by backward variable elimination under a removal criterion of significance level $p>0.1$. The covariates to be adjusted included age, sex, race, baseline BMI, sites of metastases, ECOG performance status, clear cell histology and prior lines of therapy. The HR and its 95\% CI were reported. The overall significance level was set at $\mathrm{p}<0.05$. The relationship between mGPS and survival outcomes (OS and PFS) was assessed using Kaplan-Meier analysis. Statistical analysis was conducted using SAS V.9.4, and SAS macros were developed by the Biostatistics Shared Resource at Winship Cancer Institute. ${ }^{22}$ The discrimination ability of baseline mGPS and IMDC risk score in predictive survival outcomes was measured by Uno's c-statistics. ${ }^{23}$

\section{RESULTS}

\section{Baseline demographic and disease characteristics}

We identified 156 patients who met the inclusion criteria (table 1 ). The median age was $64 ; 69 \%$ were male; and $80 \%$ were Asian or white. The majority of patients had a ECOG performance score $0(36 \%)$ or $1(49 \%)$. The predominant histology was clear cell $(78 \%)$. Seventy-five percent of patients had lung metastases, while $37 \%$ and $29 \%$ had bone and liver metastases, respectively. Most patients were receiving first-line $(38 \%)$ or second-line (44\%) immunotherapy. Fifty-seven percent of patients received a PD-1 agent as monotherapy; 29\% received combination anti-PD-1 and anticytotoxic T-lymphocyteassociated protein-4 (CTLA-4) therapy; $10 \%$ received an anti-PD-1 or anti-PD-L1 agent in combination with an antiangiogenic therapy (either a monoclonal antibody or tyrosine kinase inhibitor targeting vascular endothelial growth factor (VEGF)); and $4 \%$ were treated with an anti-PD-1 agent in addition to an experimental therapy. Baseline mGPS was 0 in 37\%, 1 in $40 \%$ and 2 in $24 \%$ of patients. When stratified according to IMDC risk category, $15 \%, 61 \%$ and $24 \%$ of the cohort were favorable, intermediate and poor risk, respectively.

\section{Baseline mGPS and survival outcomes in univariate and multivariate analyses}

Multiple variables including mGPS at baseline and their association with PFS and OS were investigated in univariate analysis (UVA) and multivariate analysis (MVA). In UVA, a baseline mGPS of 2 was associated with shorter OS 
Table 1 Patient characteristics

\begin{tabular}{lc} 
Characteristics & $\mathbf{n = 1 5 6}(\%$ or range) \\
\hline Age (years) (median) & $64(23-90)$ \\
Male gender & $108(69.2)$ \\
Race & $125(80.1)$ \\
White/Asian & $31(19.9)$ \\
Black & $78(50.0)$ \\
Ever smoker & \\
ECOG Performance Score & $56(36.4)$ \\
0 & $75(48.7)$ \\
1 & $23(14.9)$ \\
$2-3$ & \\
Histology & $118(78.1)$ \\
Clear cell & $33(21.9)$ \\
Non-clear cell & \\
Sites of metastases & $93(59.6)$ \\
Lymph node & $58(37.2)$ \\
Bone & $117(75)$ \\
Lung & $18(11.5)$ \\
Brain & $45(28.8)$ \\
Liver &
\end{tabular}

Number of prior therapies

\begin{tabular}{lc}
0 & $59(37.8)$ \\
1 & $69(44.2)$ \\
$2-6$ & $28(17.9)$ \\
\hline Therapy type & \\
Anti-PD-1 monotherapy & $89(57.1)$ \\
Anti-PD-1+anti-CTLA-4 & $45(28.8)$ \\
Anti-PD-1+anti-VEGF & $10(6.4)$ \\
Anti-PD-L1+anti-VEGF & $5(3.2)$ \\
\hline Anti-PD-1+experimental therapy & $7(4.5)$ \\
BMI (median) & $26.3(16.3-56.4)$ \\
Baseline lab values & \\
Albumin (median) & $3.75(2.0-4.7)$ \\
CRP (median) & $14.7(0.76-297.0)$ \\
Baseline mGPS & \\
\hline 0 & $57(36.6)$ \\
\hline 1 & $62(39.7)$ \\
2 & $37(23.7)$ \\
\hline IMDC risk score & $23(14.8)$ \\
Favorable & $96(61.5)$ \\
\hline Pntermediate & $37(23.7)$ \\
\hline
\end{tabular}

BMI, Body Mass Index; CRP, C reactive protein; CTLA-4, cytotoxic T-lymphocyte-associated protein-4; ECOG, Eastern Cooperative Oncology Group; IMDC, International Metastatic Renal Cell Carcinoma Database Consortium; mGPS, modified Glasgow Prognostic Score; PD-1, programmed cell death protein 1; PD-L1, programmed cell death protein ligand 1; VEGF, vascular endothelial growth factor.
(HR 4.29, CI 2.24 to 8.24, $\mathrm{p}<0.001$ ) and PFS (HR 1.90, CI 1.20 to $3.01, p=0.006$ ) relative to a score of 0 (table 2 ). The prognostic relevance of a high baseline mGPS persisted in MVA (table 3). An mGPS of 2 was associated with worse OS (HR 3.61, CI 1.78 to 7.31, $\mathrm{p}<0.001$ ) and PFS (HR 1.87, CI 1.17 to $2.97, \mathrm{p}=0.008)$. There was a trend in association of baseline mGPS of 1 and shorter OS (HR 1.82, CI 0.94 to $3.54, \mathrm{p}=0.076$ ), although this was not statistically significant.

\section{Baseline mGPS and survival outcomes in Kaplan-Meier analysis}

Median follow-up was 24.2 months. The median overall survival (mOS) of the cohort was 20.8 months $(95 \%$ CI 15.7 to not evaluable [NE]) with $66.6 \%$ of patients alive at 12 months and $48.6 \%$ at 24 months. The median progression-free survival (mPFS) was 4.4 months (95\% CI 3.5 to 6.0 ) with $26 \%$ of patients without progression at 12 months and $18.7 \%$ at 24 months.

The mOS of patients with baseline mGPS of 0,1 and 2 was 44.5 (95\% CI 27.3 to NE), 15.3 (95\% CI 11.0 to 24.2) and 10 (95\% CI 4.6 to 17.5$)$ months, respectively $(\mathrm{p}<0.0001)$ (figure 1A). The mPFS of these three cohorts was 6.7 (95\% CI 3.6 to 13.1), 4.2 (95\% CI 2.9 to 6.2$)$ and $2.6(95 \%$ CI 2.0 to 5.6) months, respectively ( $\mathrm{p}=0.0216)$ (figure 2A). The mOS stratified by IMDC risk score differed significantly $(\mathrm{p}<0.0001)$, but the mPFS did not $(\mathrm{p}=0.0728)$ (figures $1 \mathrm{~B}$ and $2 \mathrm{~B})$. The discrimination power of baseline mGPS to predict survival outcomes including OS and PFS was slightly improved compared with IMDC risk stratification based on Uno's c-statistic for each (table 3).

\section{DISCUSSION}

Immunotherapy has been fully integrated into the firstline treatment of mRCC. The IMDC and MSKCC risk scoring systems help to prognosticate before the initiation of treatment but were developed during the era of targeted therapy, involve some subjectivity as they incorporate performance status and involve multiple factors which can make them more cumbersome to use. In the largest cohort of patients with mRCC examined to date, we found the mGPS to be prognostic in patients treated with immunotherapy in any line of therapy and comparable to the IMDC risk score in its ability to predict survival outcomes.

There are multiple risk scoring systems available for prognostication in mRCC, all of which include some combination of laboratory and clinical factors such as performance status. The French model was developed by Negrier $e t a l^{24}$ based on the study of 782 patients treated with cytokine therapy (IL-2 or interferon). In their model, ECOG performance status, number of metastatic sites, time from diagnosis to metastatic disease, hemoglobin and biochemical evidence of inflammation (defined as erythrocyte sedimentation rate $>100$ or $\mathrm{CRP}>50$ ) could be used to estimate the likelihood of rapid progression while 
Table 3 Discrimination power of $\mathrm{mGPS}$ and IMDC in predicting survival outcomes based on Uno's concordance statistic

\begin{tabular}{llllll}
\hline & \multicolumn{2}{l}{ OS } & & \multicolumn{2}{l}{ PFS } \\
\cline { 2 - 3 } \cline { 5 - 6 } & Estimate & SE$^{*}$ & & Estimate & SE$^{*}$ \\
\hline mGPS & 0.6312 & 0.0435 & & 0.5752 & 0.0385 \\
IMDC & 0.6102 & 0.0397 & & 0.5533 & 0.0347 \\
\hline
\end{tabular}

*SEs are calculated using 5000 perturbation samples. IMDC, International Metastatic Renal Cell Carcinoma Database Consortium; mGPS, modified Glasgow Prognostic Score; OS, overall survival; PFS, progression-free survival.

receiving cytokine therapy. The Cleveland Clinic Foundation model was developed in 2007 via analysis of 120 patients treated with bevacizumab, sorafenib, sunitinib or axitinib during which a composite of five prognostic factors were found to predict mPFS, including corrected calcium, neutrophil count, platelet count, ECOG performance status and time from diagnosis to treatment of $<2$ years. ${ }^{25}$ The International Kidney Cancer Working Group model incorporates nine factors total to prognosticate in patients also receiving antiangiogenic therapy: four clinical factors including treatment received, KPS, number of metastatic sites, and prior immunotherapy and five lab parameters including pre-treatment hemoglobin, $\mathrm{LDH}$, alkaline phosphatase, neutrophils and calcium. ${ }^{26}$ As mentioned previously, the IMDC model is the most widely used in clinical practice and trial development, differs only slightly from the MSKCC model and reflects many of the factors incorporated into in prior models: time from diagnosis to systemic therapy, KPS performance status, calcium, and CBC parameters including hemoglobin, neutrophil and platelet counts. ${ }^{5}$ It was originally derived from a cohort of 1028 patients treated with targeted therapy and those with favorable, intermediate and poor risk scores were found to have a mOS of 43, 23 and 8 months, respectively. The IMDC model has been applied retrospectively as a prognostic tool in patients receiving second-line immunotherapy and acquired a predictive component when applied prospectively in the Checkmate214 phase III trial of ipilimumab and nivolumab versus sunitinib in mRCC. ${ }^{727}$ However, the model has multiple components and incorporates some subjectivity in the form of performance status assessment, a facet worth noting since oncologists have been shown to overestimate performance status compared with how patients view their own functioning. ${ }^{28}$ The applicability of the mGPS is intriguing given its two-factor composition, reliance solely on biomarkers and its reflection of underlying systemic inflammation, a suspected mode of resistance to immunotherapy.

There are ample data to suggest that systemic inflammation portends poorer responses to ICI across genitourinary cancers including RCC. ${ }^{11-15}{ }^{29}$ Immune checkpoint blockade inhibits the attenuation of T-cell activation centrally in the secondary lymphoid organs in the case of 


\section{A. Overall survival by mGPS}

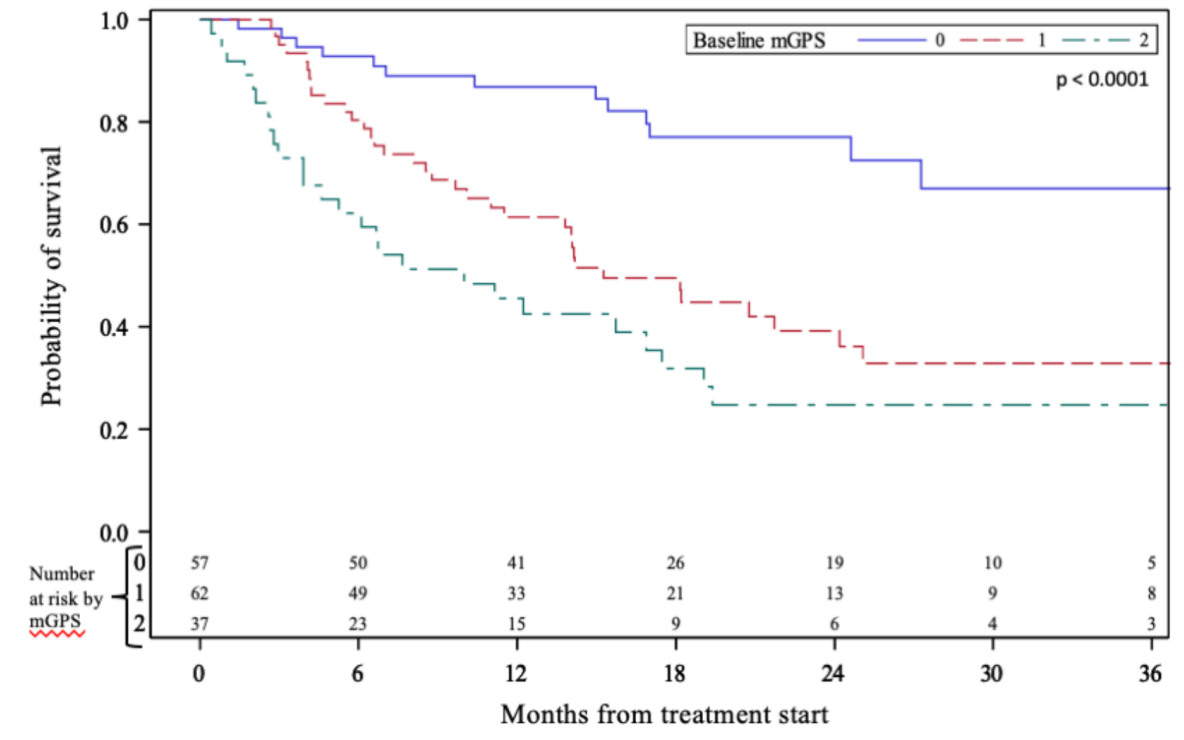

B. Overall survival by IMDC risk score

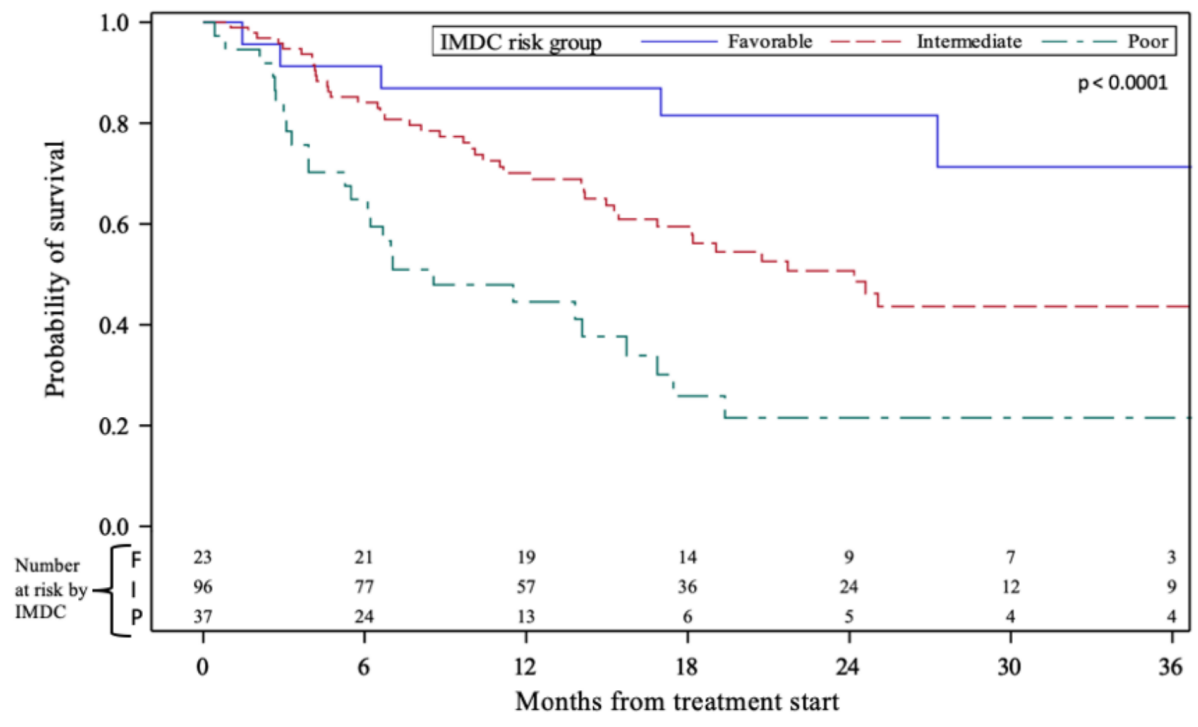

Figure 1 Kaplan-Meier estimates of (A) baseline mGPS versus (B) IMDC risk group stratification and association with overall survival. IMDC, International Metastatic Renal Cell Carcinoma Database Consortium; mGPS, modified Glasgow Prognostic Score.

anti-CTLA-4 agents and in peripheral tissues via the PD-1/ PD-L1 targeted agents. ${ }^{30}$ Intrinsic resistance to immunotherapy is felt to be related to low tumor immunogenicity and patient genetic factors affecting antigen presentation and T-cell tumor infiltration. However, outside of intrinsic tumor and host factors, an immunosuppressive milieu in the tumor microenvironment (TME) contributes as well. ${ }^{31-37}$ The combination and interactions of tumor, immune, and endothelial cells, surrounding stromal cells of the extracellular matrix and soluble chemokines and cytokines constitute the very dynamic TME. ${ }^{38}$ Adequate infiltration of a tumor by $\mathrm{T}$ cells may not be sufficient to ensure response to checkpoint inhibition if an increased number of myeloid derived suppressor cells (MDSCs) and $\mathrm{CD} 4+$ regulatory $\mathrm{T}$ (Treg) cells locally dampen the cytotoxic antitumor effects mostly through cytokine signaling. ${ }^{39}$ In metastatic urothelial carcinoma, a T-cell inflamed gene expression profile characterized by interferon gamma (IFN- $\gamma$ ) signaling predicted response to pembrolizumab in KEYNOTE052. ${ }^{40}$ However, immunosuppressive cytokines like transforming growth factor- $\beta$ and IL-10 and proinflammatory mediators like IL-6, tumor necrosis factor (TNF) and IL-1 $\beta$ simultaneously contribute to MDSC upregulation and subsequent T-cell 


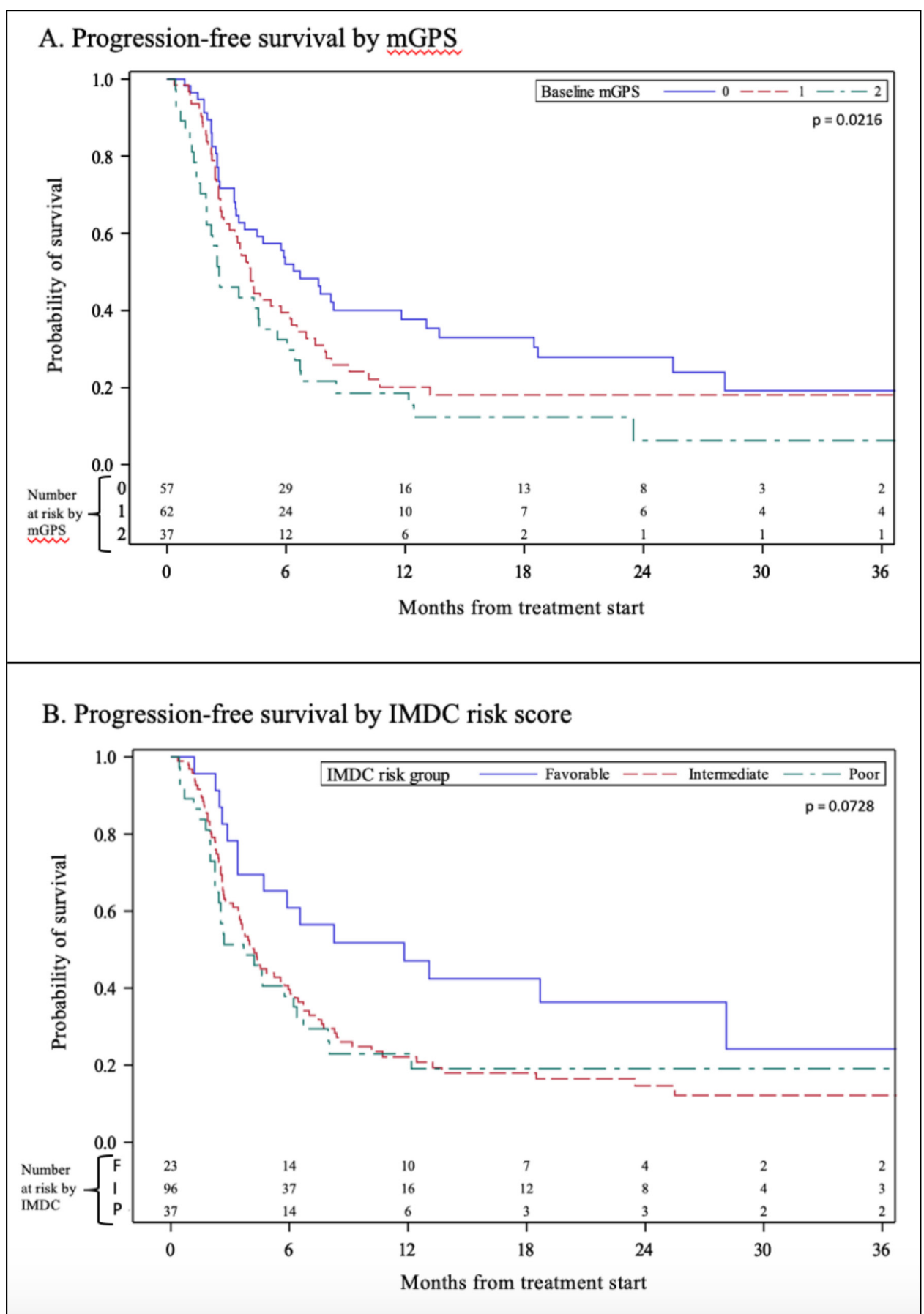

Figure 2 Kaplan-Meier estimates of (A) baseline mGPS versus (B) IMDC risk group stratification and association with progression-free survival. IMDC, International Metastatic Renal Cell Carcinoma Database Consortium; mGPS, modified Glasgow Prognostic Score.

exhaustion. ${ }^{41} 42$ Elevated levels of TNF and IL-6 are highly associated with malignancy and may further attenuate these ICI-induced cytotoxic T-cell responses. ${ }^{43}$ Depletion of intratumoral MDSCs and Tregs has been shown to potentiate PD-1/PD-L1 blockade in melanoma and breast tumors, and MDSC enrichment has been associated with lack of response to ipilimumab in patients with metastatic melanoma. ${ }^{44-46}$ Thus, chronic inflammation may contribute to tumor immune evasion at baseline but especially in the setting of ICI therapy.
The mGPS may be particularly relevant as a prognostic score in mRCC treated with ICI as it reflects some of these underlying mechanisms of immunotherapy resistance. IL-6, TNF- $\alpha$ and IL-1 $\beta$, same inflammatory cytokines that lead to MDSC upregulation and T-cell anergy, are known to lead to increased production of CRP by hepatocytes and also correlate with larger tumor size and higher stage in RCC specifically. ${ }^{47}$ Potentially in response to his angiogenic and antiapoptotic properties, elevated CRP correlates with poorer prognosis across urological 
cancers, and in both patients with localized RCC undergoing nephrectomy as well as those with metastatic disease. ${ }^{48-50}$ In fact, there are data to suggest that RCC tissue itself produces CRP in response to intratumoral IL-6 relative to adjacent normal kidney parenchyma. ${ }^{51}$ We also know that this same cytokine profile has catabolic effects and plays a role in suppressing albumin production by the liver in malignant states ${ }^{5253}$ It is not surprising then that the MGPS as the composite of these biomarkers of inflammation is capable of predicting response and survival in response to ICI. Fujiwara et al found the mGPS to be prognostic in 45 patients with mRCC treated with nivolumab in the second line. ${ }^{21}$ We have expanded on their work to include a larger cohort treated with ICI as a single agent but also in combination with another ICI, antiangiogenic therapy or a novel experimental treatment. In addition, $38 \%$ of our cohort received ICI in the first line setting. This reflects the way that ICIs are used in our current treatment paradigm and further supports the use of the mGPS as a prognostic score in this patient population.

An easy-to-use, inexpensive and accessible score capable of prognosticating in patients with mRCC receiving immunotherapy like the mGPS is valuable. Both serum assays cost about 15 dollars, equating to a sum much smaller than those typically encountered in oncological care. However, the hunt for a predictive biomarker capable of helping clinicians navigate the crowded first-line therapy space and decide on an ICI and targeted therapy combination like axitinib and pembrolizumab versus an ICI duo such as ipilimumab and nivolumab is desperately needed. The IMDC remains the only prospectively applied predictive biomarker in mRCC to date. ${ }^{7}$ While tumor PD-L1 expression has borne out in other malignancies, it is more controversial in RCC. In CHECKMATE214, among those with PD-L1 expression $\geq 1 \%$, the mPFS was 22.8 vs 5.9 months (HR $0.46,95 \%$ CI 0.31 to 0.67 ) in those who received ipilimumab plus nivolumab versus sunitinib, respectively; conversely, those with negative PD-L1 did not derive benefit from the ICI combination ( 11.0 vs 10.4 months, HR $1.00,95 \%$ CI 0.80 to 1.26 ). However, the mOS and overall response rate benefit was seen with ipilimumab plus nivolumab over sunitinib regardless of PD-L1 status. ${ }^{7}$ In the KEYNOTE426 trial of pembrolizumab plus axitinib versus sunitinib, mPFS, mOS and ORR were all improved with ICI-based therapy regardless of PD-L1 expression. ${ }^{6}$ PD-L1 does not account for the entirety of response to ICI, potentially in part due to tumor heterogeneity within a tumor, between metastatic sites and over time as well as the variable threshold definitions for positivity across different assays. Another potential biomarker is tumor mutational burden (TMB), the concept being that tumors with higher TMB are more immunogenic due to an increase in neoantigen presentation. RCC has a low average TMB of 1.1 mutations/ $\mathrm{Mb}$ compared as high as 300 mutations/ $\mathrm{Mb}$ in other cancers like melanoma, although it does have the highest frequency of notably immunogenic insertion and deletion mutations. ${ }^{545}$ In practice, TMB has not manifest as the elusive predictive biomarker to ICI in RCC. In an exploratory analysis of patients treated in IMmotion 150 with atezolizumab plus bevacizumab versus sunitinib, TMB quartile did not correlate with response. ${ }^{56}$ This same study elegantly assessed whether an angiogenic gene expression signature or an immune signature characterized by increased CD8+ T-cell infiltration, and IFN- $\gamma$ signaling could predict response to anti-VEGF and ICI therapy, respectively. A high angiogenic signature did not impact PFS in response to atezolizumab plus bevacizumab versus sunitinib or atezolizumab monotherapy versus sunitinib; however, a high immune signature correlated with increased PFS with atezolizumab plus bevacizumab over sunitinib. A biomarker capable of determining who should see ICI versus ICI in combination with anti-VEGF therapy versus anti-VEGF monotherapy in the first-line remains one of the holy grail pursuits in mRCC at this time.

There are several limitations to our findings. While our cohort tripled the size of the only other existing investigation of mGPS in mRCC, it is still a retrospective analysis that warrants external validation in a larger group of patients. We attempted to combat selection bias by examining 156 contiguous patients treated at our institution between 2015 and 2020. While our look at ICI both as monotherapy and in combination with immunotherapy and non-immunotherapy treatments reflects the realworld use of immunotherapy both in clinical trials and community practice, there is the potential for the non-ICI component of the treatment to influence the findings. In addition, we selected patients who received an ICI of any kind. Approximately $10 \%$ of our cohort received an ICI in combination with an anti-VEGF agent, but the majority of first-line options fall into this category of combination therapy; thus, our study population is not entirely representative of current practice and a future exploration of the prognostic significance of mGPS in a more homogeneous cohort receiving ICI plus anti-VEGF therapy is needed. Because we investigated ICI given in any line of therapy, it is possible that baseline lab values could have been affected by the patient's status and response after the prior treatment. The mGPS two-factor composition makes it simple and easy-to-use, but it is worth noting that the half-life of CRP and albumin are 19 hours and 21 days, respectively. A strength of the score is it dynamicity with the downside of its susceptibility to impact by acute clinical status changes. Inflammatory markers can be affected by non-malignant conditions such as sepsis, and while we collected the values within 2 weeks of ICI initiation, it was not possible to account for all confounding factors, clinically known and unknown. Lastly, we investigated prognostic relevance of the baseline, pretreatment mGPS. Thus, we did not capture how the mGPS changes during the course of treatment. There are data to suggest that post-therapy mGPS and the delta across treatment timepoints hold prognostic significance in other malignancies such as esophageal cancer, colorectal cancer and 
NSCLC. ${ }^{57-59}$ We plan to investigate the significance of mGPS over time in patients with mRCC in future analyses.

\section{CONCLUSION}

Higher baseline mGPS was found to be associated with shorter PFS and OS in patients with mRCC treated with ICI in any line of therapy. Given that the components of the score directly reflect systemic inflammation, a known factor in the presence or development of ICI resistance, it may have particular relevance as we navigate the everexpanding first-line ICI combinations. Future work is needed to determine whether mGPS is prognostic in more homogeneous cohorts, including those receiving ICI as monotherapy, in tandem with other types of immunotherapy or in combination with antiangiogenic agents. As a prognostic biomarker, the intention of the score's use is not to prevent patients from receiving immunotherapy but rather to guide expectations about response to therapy. Our findings indicate that mGPS may be a useful clinical tool and should be validated prospectively.

Twitter Jacqueline T Brown @jackiebrown_MD and Mehmet Asim Bilen @bilenma Contributors Conception/design: JTB, WBH, VAM, and MAB; provision of patients: EEH, GAR, SC, LY, SSJ, KO, BN, BCC, OK, WBH, VAM, and MAB; collection and/or assembly of data: JTB, JMS, DM, and DR; data analysis and interpretation: JTB, YL, and MAB; manuscript writing: JTB, YL, and MAB; final approval of the manuscript: JTB, YL, JMS, DM, DR, EEH, GAR, SC, LY, SSJ, HK, KO, BN, BCC, OK, WBH, VAM, and MAB.

Funding Research reported in this publication was supported in part by the Breen Foundation and National Institutes of Health/National Cancer Institute and the Biostatistics and Bioinformatics Shared Resource of the Winship Cancer Institute of Emory University under award number P30CA138292. The content is solely the responsibility of the authors and does not necessarily represent the official views of the National Institutes of Health.

Competing interests MAB has acted as a paid consultant for and/or as a member of the advisory boards of Exelixis, Bayer, BMS, Eisai, Pfizer, AstraZeneca, Janssen, Calithera Biosciences, Genomic Health, Nektar, and Sanofi, and has received grants to his institution from Xencor, Bayer, Bristol-Myers Squibb, Genentech/Roche, Seattle Genetics, Incyte, Nektar, AstraZeneca, Tricon Pharmaceuticals, Genome \& Company, AAA, Peloton Therapeutics, and Pfizer for work performed outside of the current study.

\section{Patient consent for publication Not required.}

Provenance and peer review Not commissioned; externally peer reviewed.

Data availability statement All data relevant to this retrospective study are included in the article.

Open access This is an open access article distributed in accordance with the Creative Commons Attribution Non Commercial (CC BY-NC 4.0) license, which permits others to distribute, remix, adapt, build upon this work non-commercially, and license their derivative works on different terms, provided the original work is properly cited, appropriate credit is given, any changes made indicated, and the use is non-commercial. See http://creativecommons.org/licenses/by-nc/4.0/

\section{ORCID iD}

Mehmet Asim Bilen http://orcid.org/0000-0003-4003-1103

\section{REFERENCES}

1 Siegel RL, Miller KD, Jemal A. Cancer statistics, 2020. CA Cancer J Clin 2020;70:7-30.

2 Störkel S, van den Berg E. Morphological classification of renal cancer. World J Urol 1995;13:153-8.
3 National Cancer Institute. Seer cancer STAT facts: kidney and renal pelvis cancer, 2020. Available: https://seer.cancer.gov/statfacts/html/ kidrp.html

4 Motzer RJ, Bacik J, Murphy BA, et al. Interferon-alfa as a comparative treatment for clinical trials of new therapies against advanced renal cell carcinoma. J Clin Oncol 2002;20:289-96.

5 Heng DYC, Xie W, Regan MM, et al. Prognostic factors for overall survival in patients with metastatic renal cell carcinoma treated with vascular endothelial growth factor-targeted agents: results from a large, multicenter study. J Clin Oncol 2009;27:5794-9.

6 Rini BI, Plimack ER, Stus V, et al. Pembrolizumab plus axitinib versus sunitinib for advanced renal-cell carcinoma. N Engl J Med 2019;380:1116-27.

7 Motzer RJ, Tannir NM, McDermott DF, et al. Nivolumab plus ipilimumab versus sunitinib in advanced renal-cell carcinoma. N Engl J Med 2018;378:1277-90.

8 Motzer RJ, Penkov K, Haanen J, et al. Avelumab plus axitinib versus sunitinib for advanced renal-cell carcinoma. N Engl J Med 2019;380:1103-15.

9 Choueiri TK, Powles T, Burotto M, et al. 6960 PR nivolumab + cabozantinib vs sunitinib in first-line treatment for advanced renal cell carcinoma: first results from the randomized phase III CheckMate 9ER trial. Annals of Oncology 2020;31:S1159-215.

10 Motzer R, Alekseev B, Rha S-Y, et al. Lenvatinib plus pembrolizumab or everolimus for advanced renal cell carcinoma. N Engl J Med Overseas Ed 2021;384:1289-300.

11 Martini DJ, Liu Y, Shabto JM. Novel risk scoring system for patients with metastatic renal cell carcinoma treated with immune checkpoint inhibitors. Oncologist 2019.

12 Bilen MA, Dutcher GMA, Liu Y, et al. Association between pretreatment neutrophil-to-lymphocyte ratio and outcome of patients with metastatic renal-cell carcinoma treated with nivolumab. Clin Genitourin Cancer 2018;16:e563-75.

13 Bilen MA, Martini DJ, Liu Y, et al. The prognostic and predictive impact of inflammatory biomarkers in patients who have advancedstage cancer treated with immunotherapy. Cancer 2019;125:127-34.

14 Bilen MA, Martini DJ, Liu Y, et al. Combined effect of sarcopenia and systemic inflammation on survival in patients with advanced stage cancer treated with immunotherapy. Oncologist 2020;25:e528-35.

15 Shabto JM, Martini DJ, Liu Y, et al. Novel risk group stratification for metastatic urothelial cancer patients treated with immune checkpoint inhibitors. Cancer Med 2020;9:2752-60.

16 Forrest LM, McMillan DC, McArdle CS, et al. Evaluation of cumulative prognostic scores based on the systemic inflammatory response in patients with inoperable non-small-cell lung cancer. Br J Cancer 2003;89:1028-30.

17 Proctor MJ, Morrison DS, Talwar D, et al. An inflammation-based prognostic score (mGPS) predicts cancer survival independent of tumour site: a Glasgow inflammation outcome study. Br J Cancer 2011;104:726-34.

18 Cho DS, Kim SI, Choo SH, et al. Prognostic significance of modified Glasgow prognostic score in patients with non-metastatic clear cell renal cell carcinoma. Scand J Urol 2016;50:186-91.

19 Tai CG, Johnson TV, Abbasi A, et al. External validation of the modified Glasgow prognostic score for renal cancer. Indian J Urol 2014;30:33-7.

20 Patil D, Le T-L, Bens KB, et al. Dynamic evaluation of the modified Glasgow prognostic scale in patients with resected, localized clear cell renal cell carcinoma. Urology 2020;141:101-7.

21 Fujiwara R, Takemura K, Fujiwara M, et al. Modified Glasgow prognostic score as a predictor of prognosis in metastatic renal cell carcinoma treated with nivolumab. Clin Genitourin Cancer 2020. doi:10.1016/j.clgc.2020.10.007. [Epub ahead of print: 10 Nov 2020].

22 Liu Y, Nickleach DC, Zhang C, et al. Carrying out streamlined routine data analyses with reports for observational studies: introduction to a series of generic SAS ${ }^{\circledR}$ macros. F1000Res 2018;7:1955.

23 Uno H, Cai T, Pencina MJ, et al. On the C-statistics for evaluating overall adequacy of risk prediction procedures with censored survival data. Stat Med 2011;30:1105-17.

24 Négrier S, Escudier B, Gomez F, et al. Prognostic factors of survival and rapid progression in 782 patients with metastatic renal carcinomas treated by cytokines: a report from the Groupe Français d'Immunothérapie. Ann Oncol 2002;13:1460-8.

25 Choueiri TK, Garcia JA, Elson P, et al. Clinical factors associated with outcome in patients with metastatic clear-cell renal cell carcinoma treated with vascular endothelial growth factor-targeted therapy. Cancer 2007;110:543-50.

26 Manola J, Royston P, Elson P, et al. Prognostic model for survival in patients with metastatic renal cell carcinoma: results from the International kidney cancer Working group. Clin Cancer Res 2011;17:5443-50. 
27 Yip S, Wells C, Moreira RB, et al. Real world experience of immunooncology agents in metastatic renal cell carcinoma: results from the IMDC. JCO 2017;35:492

28 Bergerot CD, Philip EJ, Bergerot PG, et al. Discrepancies between genitourinary cancer patients' and clinicians' characterization of the eastern cooperative Oncology Group performance status. Cancer 2021;127:354-8.

29 Brown JT, Liu Y, Shabto JM, et al. Baseline modified Glasgow prognostic score associated with survival in metastatic urothelia carcinoma treated with immune checkpoint inhibitors. Oncologist 2021;26:397-405.

30 Wei SC, Duffy CR, Allison JP. Fundamental mechanisms of immune checkpoint blockade therapy. Cancer Discov 2018;8:1069-86.

31 Zhao X, Subramanian S. Intrinsic resistance of solid tumors to immune checkpoint blockade therapy. Cancer Res 2017;77:817-22.

32 Pitt JM, Vétizou M, Daillère R, et al. Resistance mechanisms to Immune-Checkpoint blockade in cancer: tumor-intrinsic and -Extrinsic factors. Immunity 2016;44:1255-69.

33 Vajaitu C, Draghici CC, Solomon I, et al. The central role of inflammation associated with checkpoint inhibitor treatments. $J$ Immunol Res 2018;2018:1-10.

34 Barrueto L, Caminero F, Cash L, et al. Resistance to checkpoint inhibition in cancer immunotherapy. Transl Oncol 2020;13:100738

35 Jenkins RW, Barbie DA, Flaherty KT. Mechanisms of resistance to immune checkpoint inhibitors. Br J Cancer 2018;118:9-16.

36 Nowicki TS, Hu-Lieskovan S, Ribas A. Mechanisms of resistance to PD-1 and PD-L1 blockade. Cancer J 2018;24:47-53.

37 Sharma P, Hu-Lieskovan S, Wargo JA, et al. Primary, adaptive, and acquired resistance to cancer immunotherapy. Cell 2017;168:707-23.

38 Anderson NM, Simon MC. The tumor microenvironment. Curr Biol 2020;30:R921-5.

39 Labani-Motlagh A, Ashja-Mahdavi M, Loskog A. The tumor microenvironment: a milieu hindering and obstructing antitumor immune responses. Front Immunol 2020;11:940.

40 O'Donnell PH, Grivas P, Balar AV, et al. Biomarker findings and mature clinical results from KEYNOTE-052: first-line pembrolizumab (pembro) in cisplatin-ineligible advanced urothelial cancer (UC). JCO 2017;35:4502.

41 Wherry EJ, Kurachi M. Molecular and cellular insights into T cell exhaustion. Nat Rev Immunol 2015;15:486-99.

42 Ostrand-Rosenberg S, Sinha P. Myeloid-Derived suppressor cells: linking inflammation and cancer. J Immunol 2009;182:4499-506.

43 Zhao X, Rong L, Zhao X, et al. Tnf signaling drives myeloid-derived suppressor cell accumulation. J Clin Invest 2012;122:4094-104.

44 Steinberg SM, Shabaneh TB, Zhang P, et al. Myeloid cells that impair immunotherapy are restored in melanomas with acquired resistance to BRAF inhibitors. Cancer Res 2017;77:1599-610.
45 Taylor NA, Vick SC, Iglesia MD, et al. Treg depletion potentiates checkpoint inhibition in claudin-low breast cancer. J Clin Invest 2017;127:3472-83.

46 Meyer C, Cagnon L, Costa-Nunes CM, et al. Frequencies of circulating MDSC correlate with clinical outcome of melanoma patients treated with ipilimumab. Cancer Immunol Immunother 2014:63:247-57.

47 Yoshida N, Ikemoto S, Narita K, et al. Interleukin-6, tumour necrosis factor alpha and interleukin-1beta in patients with renal cell carcinoma. Br J Cancer 2002;86:1396-400.

48 Zhou L, Cai X, Liu Q, et al. Prognostic role of C-reactive protein in urological cancers: a meta-analysis. Sci Rep 2015;5:12733.

49 Nagasaki T, Hara M, Nakanishi H, et al. Interleukin-6 released by colon cancer-associated fibroblasts is critical for tumour angiogenesis: anti-interleukin- 6 receptor antibody suppressed angiogenesis and inhibited tumour-stroma interaction. $\mathrm{Br} \mathrm{J}$ Cancer 2014;110:469-78.

50 Gadó K, Domján G, Hegyesi H, et al. Role of interleukin-6 in the pathogenesis of multiple myeloma. Cell Biol Int 2000;24:195-209.

51 Jabs WJ, Busse M, Krüger S, et al. Expression of C-reactive protein by renal cell carcinomas and unaffected surrounding renal tissue. Kidney Int 2005;68:2103-10.

52 McMillan DC, Watson WS, O'Gorman P, et al. Albumin concentrations are primarily determined by the body cell mass and the systemic inflammatory response in cancer patients with weight loss. Nutr Cancer 2001;39:210-3.

53 Gupta D, Lis CG. Pretreatment serum albumin as a predictor of cancer survival: a systematic review of the epidemiological literature. Nutr J 2010;9:69.

54 de Velasco G, Miao D, Voss MH, et al. Tumor mutational load and immune parameters across metastatic renal cell carcinoma risk groups. Cancer Immunol Res 2016;4:820-2.

55 Alexandrov LB, Nik-Zainal S, Wedge DC, et al. Signatures of mutational processes in human cancer. Nature 2013;500:415-21.

56 McDermott DF, Huseni MA, Atkins MB, et al. Clinical activity and molecular correlates of response to atezolizumab alone or in combination with bevacizumab versus sunitinib in renal cell carcinoma. Nat Med 2018;24:749-57.

57 Kasahara N, Sunaga N, Tsukagoshi Y, et al. Post-Treatment Glasgow prognostic score predicts efficacy in advanced non-small-cell lung cancer treated with anti-PD1. Anticancer Res 2019;39:1455.

58 Guthrie GJK, Roxburgh CSD, Farhan-Alanie OM, et al. Comparison of the prognostic value of longitudinal measurements of systemic inflammation in patients undergoing curative resection of colorectal cancer. Br J Cancer 2013;109:24-8.

59 Jomrich G, Hollenstein M, John M, et al. The modified Glasgow prognostic score is an independent prognostic indicator in neoadjuvantly treated adenocarcinoma of the esophagogastric junction. Oncotarget 2018;9:6968-76. 